\title{
PSEUDOMOMENTS FOR GENERALIZED DOMAINS OF ATTRACTION
}

\author{
MARK M. MEERSCHAERT
}

(Communicated by Andrew M. Bruckner)

\begin{abstract}
In this paper we resolve a conjecture of Weiner (1987) concerning the existence of the so-called pseudomoments for a random vector which belongs to the generalized domain of attraction of an operator-stable law. The proof is a direct consequence of the spectral decomposition theorem for generalized domains of attraction in Meerschaert (1991).
\end{abstract}

\section{INTRODUCTION}

Let $\mu, \nu$ denote probability distributions on $\mathbb{R}^{k}$, and suppose that $\nu$ is nondegenerate (i.e. suppose $\nu$ cannot be supported on any $k-1$ dimensional affine subspace of $\mathbb{R}^{k}$ ). Let $\left\{X_{n}\right\}$ be independent random vectors on $\mathbb{R}^{k}$, identically distributed according to $\mu$, and let $Y$ denote a random vector on $\mathbb{R}^{k}$ with distribution $\nu$. We say that $\mu$ belongs to the generalized domain of attraction of $\nu$ if there exist linear operators $A_{n}$ on $\mathbb{R}^{k}$ and constants $b_{n} \in \mathbb{R}^{k}$ such that

$$
A_{n}\left(X_{1}+\cdots+X_{n}\right)-b_{n} \Rightarrow Y .
$$

Generalized domains of attraction were introduced in Hahn and Klass (1980), which also contains a characterization of the generalized domain of attraction of a normal law.

Suppose $\left\{Y_{n}\right\}$ are independent and identically distributed according to $\nu$ nondegenerate. We say that $\nu$ is operator-stable if for all $n=1,2,3, \ldots$ there exist linear operators $A_{n}$ and constants $a_{n}$ such that $A_{n}\left(Y_{1}+\cdots+Y_{n}\right)-a_{n}$ again has the distribution $\nu$. Operator-stable laws were characterized by Sharpe (1969), who showed that the operator-stable laws on $\mathbb{R}^{k}$ are the only possible nondegenerate limits which can occur in (1.1) above. Hahn and Klass (1985) provided the first characterization of generalized domains of attraction for the case of a general operator-stable limit.

Received by the editors April 13, 1990.

1980 Mathematics Subject Classification (1985 Revision). Primary 60F05.

Key words and phrases. Moments, operator-stable, generalized domains of attraction, spectral decomposition theorem. 
Operator-stable laws and generalized domains of attraction are a natural generalization of stable laws and domains of attraction in $\mathbb{R}^{1}$. In the onedimensional case, Feller (1971) provided an elegant description of the moment behavior of stable laws and domains of attraction using the tools of regular variation. The extension of these results to $\mathbb{R}^{k}$ was made more difficult due to the fact that the linear operators $A_{n}$ in (1.1) above many contain rotations as well as scaling. Complete results for operator-stable laws are contained in Meerschaert (1990). Moment results for generalized domains of attraction appeared in Meerschaert (1991), along with an alternative characterization of the generalized domain of attraction of an operator-stable law based on multivariate regular variation. These results are directly analogous to the one-dimensional case as described in Feller (1971).

Associated with every operator-stable law $\nu$ there is a (not necessarily unique) linear operator $B$ on $\mathbb{R}^{k}$ called an exponent of $\nu$ which plays the same role as the characteristic exponent of a one-dimensional stable law. If $\left\{Y_{n}\right\}$ are i.i.d. as before then $n^{-B}\left(Y_{1}+\cdots+Y_{n}\right)-a_{n}$ has distribution $\nu$ for all $n$, for some $a_{n} \in \mathbb{R}^{k}$. Here $n^{-B}=\exp (B \log n)$ where exp is the exponential operator defined by $\exp (A)=I+A+A^{2} / 2 !+A^{3} / 3 !+\cdots$. The path behavior of $t^{B}$ can best be understood as a reparametrization $[\log t=s]$ of the general solution to a linear system of ordinary differential equations. The norming operators $n^{-B}$ may perform rotations as well as scaling. In order to incorpcrate this geometry into the study of moment behavior for operator-stable laws and generalized domains of attraction, Weiner (1987) introduced the concept of pseudomoments.

Given a nondegenerate random vector $Z$ defined on a finite dimensional Euclidean space, and a nonsingular linear operator $A$ on the same space, the $A$-pseudomoment of order $p$ of $Z$ is defined by

$$
E(Z, A, p)=\int_{0}^{\infty} \operatorname{Pr}\left[\left\|t^{-A / p} Z\right\| \geq 1\right] d t .
$$

For $Y$ operator-stable with exponent $B$, Weiner showed that $E(Y, B, p)<\infty$ for all $0<p<1$. If $Y$ is Gaussian then $E(Y, B, p)<\infty$ for all $p>0$, and otherwise $E(Y, B, p)=\infty$ for $p \geq 1$.

Now if (1.1) holds in the special case $A_{n}=n^{-B}$ for all $n$, where $B$ is an exponent of $\nu$, we say that $\mu$ belongs to the domain of normal attraction of $\nu$. Weiner showed that for $X$ in the domain of normal attraction of $Y$, the pseudomoment $E(X, B, p)<\infty$ for $0<p<1$, and for $p \geq 1$ the pseudomoment converges in the case of a normal limit $Y$ and diverges otherwise. Weiner goes on to conjecture that the same results hold (suitably modified in the case $p=1$ ) when $X$ belongs to the generalized domain of attraction of $Y$.

The purpose of this paper is to resolve Weiner's conjecture. The conjecture as it stands is false, due to the fact that the norming operators in (1.1) can contain rotations which are not allowed in the special case of normal attraction. However, it is true that similar pseudomoment results can be obtained for any $X$ in the generalized domain of attraction of an operator-stable law after an appro- 
priate rotation. In the next section we will provide a corrected statement of the general pseudomoment results, based on the spectral decomposition theorem of Meerschaert (1991).

\section{RESULts}

As before, let $\left\{X_{n}\right\}$ denote i.i.d. random vectors on $\mathbb{R}^{k}$ with distribution $\mu$, and let $Y$ denote a nondegenerate random vector on $\mathbb{R}^{k}$ with operator-stable distribution $\nu$. Associated with any operator-stable law $\nu$ there is a direct sum decomposition $\mathbb{R}^{k}=V_{1} \oplus \cdots \oplus V_{m}$ called the spectral decomposition, introduced in Meerschaert (1990). If $B$ is any exponent of $\nu$, let $\lambda_{1} \cdots \lambda_{m}$ denote the real spectrum of $B$, i.e. the real parts of eigenvalues of $B$. Then $V_{i}$ is the direct sum of the generalized eigenspaces of $B$ for eigenvalues with real part equal to $\lambda_{i}$. Since $V_{1} \cdots V_{n}$ are $B$-invariant subspaces it is obvious that they are also $n^{-B}$ invariant.

If (1.1) holds for some sequence of norming operators $A_{n}$ having the property that $V_{1} \cdots V_{m}$ are $A_{n}$-invariant subspaces for all $n$, we say that $\mu$ is spectrally compatible with $\nu$. If $\mu$ belongs to the domain of normal attraction of $\nu$, then obviously $\mu$ is spectrally compatible with $\nu$. This is not true in the case of generalized domains of attraction; however the spectral decomposition theorem of Meerschaert (1991) states that for every $\mu$ in the generalized domain of attraction of $\nu$ operator-stable, there exists a linear operator $T$ on $\mathbb{R}^{k}$ such that $T \mu$ is spectrally compatible with $\nu$. The linear operator $T$ is simply a change of coordinates, and so we may take $T$ to be orthogonal.

Theorem. If $\mu$ is spectrally compatible with $\nu$ then:

(i) $E\left(X_{n}, B, p\right)<\infty$ for all $0<p<1$;

(ii) if $\nu$ has a nonnormal component then $E\left(X_{n}, B, p\right)=\infty$ for all $p>1$.

If $p=1$, or if $p>1$ and $\nu$ is purely Gaussian, then the pseudomoment will be finite for some $\mu$ and infinite for some others. The choice of exponent $B$ does not alter the results of the theorem; however, it does effect the numerical value of the pseudomoment in the case of convergence. By virtue of the spectral decomposition theorem, we obtain the following result, which characterizes pseudomoments for generalized domains of attraction.

Corollary. If $\mu$ belongs to the generalized domain of attraction of $\nu$ then there exists an orthogonal transformation $T$ on $\mathbb{R}^{k}$ such that:

(i) $E\left(T X_{n}, B, p\right)<\infty$ for all $0<p<1$;

(ii) if $\nu$ has a nonnormal component then $E\left(T X_{n}, B, p\right)=\infty$ for all $p>1$.

We remark that in the case of $\nu$ normal, every $\mu$ in the generalized domain of attraction of $\nu$ is automatically spectrally compatible since the spectral decomposition of $\nu$ consists of a single component. 


\section{Proof of the Theorem}

Suppose that $\nu$ is operator-stable on $\mathbb{R}^{k}$ and $B$ is an exponent of $\nu$. Sharpe (1969) shows that the real spectrum $\lambda_{1} \cdots \lambda_{m}$ of $B$ is contained in the interval $[1 / 2, \infty)$. If $\mu$ belongs to the generalized domain of attraction of $\nu$, and if $\mu$ is spectrally compatible with $\nu$, then by Theorem 4.1 of Meerschaert (1991) we have that

$$
\log \left\|A_{n} x\right\| / \log n \rightarrow-\lambda_{i}
$$

for all $x \neq 0$ in $V_{i}$, where $V_{1} \cdots V_{m}$ is as before the spectral decomposition relative to $\nu$. In other words $n^{p}\left\|A_{n} x\right\| \rightarrow 0$ for $0<p<\lambda_{i}$ and $n^{p}\left\|A_{n} x\right\| \rightarrow \infty$ for $p>\lambda_{i}$. A computation shows that for $x \in V_{i}$

$$
\log \left\|t^{B} x\right\| / \log t \rightarrow \lambda_{i}
$$

as $t \rightarrow \infty$ for all $x \neq 0$ in $V_{i}$. See, for example, Meerschaert (1990).

Now if (1.1) holds, then by the standard convergence criteria for triangular arrays we have $n \mu\left\{A_{n}^{-1} d x\right\} \rightarrow \phi\{d x\}$, where $\phi$ is the Lévy measure of the limit distribution $\nu$. Let $H$ denote the $\phi$-continuity set $\{x:\|x\| \geq 1\}$. The $B$-pseudomoment of order $p$ of $X_{n}$ is

$$
\begin{aligned}
\int_{0}^{\infty} & \operatorname{Pr}\left[\left\|t^{-B / p} X_{n}\right\| \geq 1\right] d t \\
& =\int_{0}^{\infty} \operatorname{Pr}\left[\left\|s^{-B} X_{n}\right\| \geq 1\right] p s^{p-1} d s \\
& =\int_{0}^{\infty} \mu\left(s^{B} H\right) p s^{p-1} d s .
\end{aligned}
$$

By the above arguments we have that $\left\|A_{n} n^{(1+\varepsilon) B}\right\| \rightarrow \infty$ and $\left\|A_{n} n^{(1-\varepsilon) B}\right\| \rightarrow 0$ uniformly on compact sets for all $\varepsilon>0$ small. Thus as $n \rightarrow \infty$, we have $n \mu\left(n^{(1+\varepsilon) B} H\right)=n \mu\left(A_{n}^{-1} A_{n} n^{(1+\varepsilon) B} H\right) \rightarrow 0$ and $n \mu\left(n^{(1-\varepsilon) B} H\right) \rightarrow \infty$ (assuming that $\nu$ is nonnormal, i.e. that $\phi$ is not the zero measure). By a change of variable, we have $n^{1+\delta} \mu\left(n^{B} H\right) \rightarrow \infty$ and $n^{1-\delta} \mu\left(n^{B} H\right) \rightarrow 0$ for all $\delta>0$ small, or in other words,

$$
\log \mu\left(n^{B} H\right) / \log n \rightarrow 1 .
$$

By an easy continuity argument, the same holds with $n$ replaced by $t$ real.

Now if $p>1$ and $\nu$ is nonnormal, then (choose $\delta<p-1$ ) for some $s_{0}>0$ we have $\mu\left(s^{B} H\right) \geq s^{1-\delta}$ for all $s \geq s_{0}$ and so the pseudomoment is infinite. If $0<p<1$ then (choose $\delta<1-p$ ) we have $\mu\left(s^{B} H\right) \geq s^{1+\delta}$ for all large $s$, and so the pseudomoment converges. This concludes the proof.

\section{REFERENCES}

Araujo, A. and E. Gine (1980), The central limit theorem for real and Banach valued random variables, Wiley, New York.

Feller, W. (1971), An introduction to probability theory and its applications, Wiley, New York. 
Hahn, M. and M. Klass (1980), Matrix normalization of sums of random vectors in the domain of attraction of the multivariate normal, Ann. Probab. 8, 262-280.

Hahn, M. and M. Klass (1985), Affine normability of partial sums of I.I.D. random vectors: $A$ characterization, Z. Wahrsch. Verw. Geb. 69, 479-505.

Meerschaert, M. (1991), Spectral decomposition for generalized domains of attraction, Ann. Probab. 19.

, (1990), Moments of random vectors which belong to some domain of normal attraction, Ann. Probab. 18, 870-876.

Sharpe, M. (1969), Operator-stable probability distributions on vector groups, Trans. Amer. Math. Soc. 136, 51-65.

Weiner, D. (1987), On the existence and convergence of pseudomoments for variables in the domain of normal attraction of an operator-stable distribution, Proc. Amer. Math. Soc. 101, 521-528.

Department of Mathematics, Albion College, Albion, Michigan 49224 